\title{
SERVOMOTOR BASED ELECTRONIC STEERING SYSTEM IN FOUR-WHEELER VEHICLES BY MEANS OF AN ACCELEROMETER
}

\author{
Ronauk Kumar Maharana \\ B. Tech, Mechanical Engineering, College of Engineering and Technology, Bhubaneswar, Odisha, India
}

\begin{abstract}
In Olden Days Steering Mechanisms Developed To Turn A Vehicle At A Certain Radius While Negotiating A Turn Had A Greater Steering Gear Ratio. For A Small Rotation Of The Steering Gear The Steering Wheel Had To Be Given A Greater Effort For Larger Degree Of Rotation Than That Of The Steering Gear Inorder To Develop Torque For Turning The Vehicle. To Reduce The Effort At The Steering Wheel, Power Steering Was Developed In The $18^{\text {th }}$ Century. It Not Only Made Steering Easier But Also Increased The Accuracy In Steering A Four Wheeled Vehicle. Precision Turning In Four Wheeled Vehicles Not Only Prevents The Vehicle From Skidding But Also Prevents Road Accidents. Various Other Mechanisms Were Also Established For Reducing The Steering Effort And Precision Turning Including Hydraulics As Well As Electronic Type. Taking Into Account The Various Systems Of Steering As Well As Using A Bit Of Electronics And Microcontroller Application A Modern Technique Can Be Developed Using A Servomotor, An Accelerometer And A Microprocessor For An Increased Precision In Steering Of A Vehicle Which May Be Termed As The "Accelerometer Driven Servomotor Based Steering Gear" Or "A.S. Steering Gear". In This Paper, I Shall Discuss How To Use An Accelerometer To Control A Servomotor Inorder To Help In Steering Of A Vehicle By Means Of Basic Microcontroller Applications And Programming.
\end{abstract}

Keywords - Servomotor, Accelerometer, Fundamental Law Of Correct Gearing, Steering Gear Ratio, Microprocessor, Analog To Digital Conversion, Pulse Width Modulation, Rack And Pinion Assembly

\section{INTRODUCTION}

Whenever there is a requirement in change in direction of a moving vehicle the steering mechanism comes in action. The steering of a four-wheeled vehicle is made inorder to roll the front wheels without any lateral slip. The front wheels are supported on two stub axles so that they can turn left or right for steering. This is done by mechanical linkages and gears between the steering wheel infront of the driver and the steering gear cross shaft. The complete arrangement is called the steering system. The function of the steering system is to convert the rotation caused by steering wheel into angular deviation of the front wheels. Many modern cars use rack and pinion steering gear, where the steering wheel turns the pinion which moves the rack with which it is meshed. So, circular motion is converted to linear motion along the transverse axis of the car. A large degree of feedback and direct steering feel is produced in this type of steering gear. A disadvantage is that it is not adjustable, so when the assembly wears and lashes, it has to be replaced only.

In many vehicles, generally the heavy duty ones, a large steering effort was needed to be produced at the steering wheel for a heavy torque to turn the vehicle. The steering gear ratio i.e. the number of turns on the steering wheel required to produce one turn of the steering gear wheel becomes very high for these vehicles. To overcome this and reduce the steering gear ratio, the power steering was developed to be installed and used in modern day vehicles. The power steering helped the driver by reducing the effort at the steering wheel making it smooth for driving. Hydraulic or electric actuators add controlled energy to the steering mechanism, so that less effort is given by driver for turning the wheels while driving at typical speeds, and reduce the high effort required to turn the wheels when a vehicle is stopped or moving slowly. A servomotor can be used as an actuator for the purpose of power steering. The shaft of the servomotor can be coupled to the steering gear wheel to which the pitman arm is attached and thus the servo can be used as an actuator for power steering purpose by means of any servo controller. Hence the servomotor can provide effective steering while driving a vehicle.

\section{AUTOMOBILE STEERING GEARS}

When a four wheeler makes a turn towards one particular side, the front wheel of that side must swing about the pin through a greater angle than the wheel of the other side. The ideal relation between the swings of the two wheels would be if the axes of the stub axles intersect at a point on the common axis of the two rear wheels. All the wheels of the vehicle will move about a vertical axis at that point thus minimising the tendency of the wheels to skid.

If $\varphi$ and $\Theta$ are taken to be the angles turned by the outer and inner wheels respectively then a relation comes out as: $\operatorname{Cot} \varphi-\operatorname{Cot} \theta=\frac{\mathrm{a}}{\mathrm{b}}$ 
Where ' $a$ ' is the length between the pivots of front axles and ' $b$ ' is the length of wheel base.

The above equation is known as "Fundamental equation for Correct Gearing" and the mechanisms which fulfil this fundamental equation are known as "Steering Gear Mechanisms".

Basically in an automobile there are two modes of steering gear mechanisms viz. Davis steering gear and Ackermann steering gear. A Davis steering gear has sliding pairs and the gear fulfils the fundamental equation of gearing in all positions. An Ackermann steering gear has turning pairs and fulfils the fundamental equation at the middle and two extreme positions but not everywhere. Apart from these classifications the steering gears are classified on the basis of the design of steering gearbox such as Worm and sector steering gear, Cam and roller steering gear, Rack and pinion steering gear, Cam and double lever steering gear, etc. Out of these the most common and widely used steering gear is the rack and pinion steering gear, driven by the rack and pinion gear mechanism. In this mechanism the movement of pinion gear is done by the help of the steering wheel in the hands of the driver. The pinion drives the rack and by that means the rack helps in movement of the stub axles by means of the knuckle arm. The A.S. steering gear also uses a rack and pinion mechanism where the difference lies at the point that there is no mechanical contact between the steering wheel and the steering gear cross shaft.

\section{DESCRIPTION OF THE MECHANISM}

The Accelerometer driven Servomotor based steering gear mechanism or simply the A.S. steering gear consists of a rack and pinion gear mechanism in which a servomotor drives the pinion or the steering gear wheel. A servomotor is a special kind of motor that develops precise control of angular or linear positions, velocity and acceleration. It consists of a suitable motor coupled to a sensor for position feedback. By means of coupling the shaft of the servomotor is coupled to the steering gear wheel and thus precise motion of the shaft makes controlled rotation of the steering gear cross shaft. A servomotor also requires a servo controller which is a dedicated module designed specifically for its use. For that purpose an accelerometer is attached to the steering wheel which controls the movement of the motor. The overall control of the mechanism is performed by means an electronic control unit (ECU) which may be a microcontroller or a microprocessor that works over analog to digital conversion (ADC). The accelerometer is rotated by means of the steering wheel that transmits the analog data to the ECU where analog to digital conversion takes place. The digital data is manipulated as electric current, required for driving the servomotor. For a certain rotation of the accelerometer there is a précised rotation of shaft and thus the pinion makes the rack to undergo linear motion. Hence steering movement takes place. However there is no mechanical contact between the steering wheel and the steering gear cross shaft which is unique and quite advantageous for an automobile in terms of space and volume reduction.

\section{MACHINE COMPONENTS USED FOR THE MECHANISM}

3.1 Steering Wheel: The purpose of steering wheel is to assist the driver inorder to take a turn while driving a vehicle. It aims at reducing the friction while steering and offers the driver to produce less effort. Two wheeler automobiles come with handle rather than steering since only one of the wheels needs to be turned. But for a four wheeler vehicle a steering mechanism is provided where the front two wheels are turned for changing the direction of the vehicle where the input is provided from the steering wheel provided to the driver.

3.2 Accelerometer: An accelerometer is a Micro Electro Mechanical System (MEMS) based device that measures proper acceleration i.e. the acceleration due to gravity in the three dimensional $\mathrm{X}, \mathrm{Y}$ and $\mathrm{Z}$ axes and computes the output in form of electric voltage. They possess a wide range of applications in various industries. In this mechanism an accelerometer is attached to the steering wheel where one axis is fixed that coincides with the axis of the steering wheel. Hence by rotating the accelerometer about that axis will provide an electric voltage corresponding to the direction desired for negotiating a turn with the steering wheel.

3.3 Microprocessor: A microprocessor is a micro-computer that that incorporates the functions of a computer's central processing unit on a single integrated circuit (IC), or at most a few ICs. Many different varieties of microprocessors are available for user-friendly purposes and possess a wide range of applications around the globe. A microprocessor can perform analog to digital conversion, for which it can interpret the signals provided by the accelerometer to control the motor.

3.4 Servomotor: A servomotor is a special type of motor that develops precise control of angular or linear positions, velocity and acceleration. It consists of a suitable motor along with a sensor for position feedback. It also requires a servo controlling module specially built for the purpose of controlling the motion of the shaft. The servomotor obtains signals through its channel from the microprocessor such that it is able to rotate its shaft whenever there is a potential difference generated due to the rotational movement of the steering wheel.

3.5 Rack and Pinion assembly: The shaft of the servomotor is coupled to a pinion gear of a rack and pinion assembly provided for steering in an automobile. As usual the pinion is always considered as the driving gear. For a rotational motion of the pinion there is linear motion of the rack. As a result due to rotation of shaft, the rack translates resulting in application of force over the knuckle arm.

3.6 Knuckle arm: Two knuckle arms are provided at the two stub axles over which the wheels are mounted. The middle eyes of both the knuckle arms are hinged at each end of the rack. Hence, as a result of translating motion of the 
rack, the knuckle arms cause the stub axles of both the wheels to turn about a common instantaneous centre. One arm suffers push at the eye while the other suffers pull. As a result steering of the four-wheeled vehicle takes place by this action.

\section{CONTROLLING A SERVOMOTOR USING AN ACCELEROMETER}

An accelerometer is a MEMS device that measures proper acceleration i.e. the acceleration due to gravity along the three dimensional $\mathrm{X}, \mathrm{Y}$ and $\mathrm{Z}$ axes and computes the output in form of electric voltage. Even though the output is in form of electric voltage but the signals are interpreted in form of analog data or analog signals. Since accelerometer is an electronic device it works over the voltage range 0 to 5 volts. For any analog device, signalling of data is done in a very specialized manner where a predefined numerical value is set for a particular voltage. The numerical value set for 5 volts is 1024 . So for a voltage value of ' $v$ ' volts the numerical value is given by,

$\mathrm{n}=(\mathbf{1 0 2 4} \times(\mathbf{v} / \mathbf{5}))$

The analog data is fed to the microcontroller in form of these numerical values and the microcontroller performs analog to digital conversion (ADC). The microcontroller manipulates the analog data and produces digital output in for of voltages. In certain microcontrollers pulse width modulation (PWM) can also be performed. Hence the analog data can be directly interpreted to generate PWM outputs. The PWM outputs are obtained in for of varying voltages which are necessary for driving of devices like brushless (BLDC) motors, servomotors. Thus the varying voltage generated becomes the input for a servomotor.

In a servomotor the shaft rotates for a specified angle and not complete rotation. For a unit value of voltage the shaft rotates for a specific angle. A servomotor, unlike the normal DC motor, possesses three wires of different colours and functions viz. positive voltage wire (red), ground wire (black, sometimes brown), signal wire (yellow). The positive voltage is the actual voltage supplied by the battery to the motor. But signal wire supplies only that amount of voltage to run the motor which is interpreted by the microcontroller on the basis of the coding performed. So the applied voltage for the servomotor will be the required amount of voltage supplied by the signal wire. As mentioned earlier, a servomotor requires a specified module or a controller for its operation, so an accelerometer along with a microcontroller can solve its problem. The function of a servomotor controller is to provide a range of voltages for the motor to turn an angle for each voltage value. For a motor we know that the applied voltage is given by,

$\mathbf{V}=\mathbf{E}_{\mathbf{b}}+\mathbf{I}_{\mathbf{a}} \mathbf{R}_{\mathbf{a}}$ where, $\mathrm{E}_{\mathrm{b}}$ is the back E.M.F., $\mathbf{R}_{\mathrm{a}}$ is the armature resistance and $\mathrm{I}_{\mathrm{a}}$ is the armature current.

When a specific voltage is applied over the motor the motor rotates and the speed is measured in terms of revolutions per minute (RPM). The RPM of the motor computes the angle turned by the shaft. The speed $\mathrm{N}$ of the motor (in terms of
RPM) is obtained from the equation,

$\mathbf{E}_{\mathrm{b}}=(\varphi \mathrm{ZN} / 60) \times(\mathbf{P} / \mathrm{A}) \quad$ where, $\mathrm{E}_{\mathrm{b}}$ is the back E.M.F., $\varphi$ is the magnetic flux, $\mathrm{Z}$ is the number of conductors, $\mathrm{P}$ is the number of poles and $\mathrm{A}$ is the number of parallel paths.

Keeping the values of $\varphi, Z, P$ and A constants, the value of $\mathrm{N}$ can be obtained just by varying the applied voltage since the back E.M.F. is dependent over the applied E.M.F. Now once the speed $\mathrm{N}$ is obtained for the motor the angle turned by the shaft can be found out.

For $\mathrm{N}$ rotations the time taken is one minute.

So, for one rotation the time taken is $(1 / \mathrm{N})$ minutes i.e. $(60 / \mathrm{N})$ seconds.

So, for $2 \pi$ angle turned (since one rotation) the time taken is $(60 / \mathrm{N})$ seconds.

For 1 radian angle turned the time taken is $(60 / 2 \pi \mathrm{N})$ seconds.

For $1^{0}$ angle turned the time taken is $((60 / 2 \pi \mathrm{N}) \times(180 / \pi))=$ $\left(5400 / \pi^{2} \mathrm{~N}\right)$ seconds.

Hence for $\Theta^{0}$ angle turned the time taken will be $((5400 \mathrm{x}$ $\Theta) / \pi^{2} N$ ) seconds.

From the above logic we can find that the time taken for rotation of shaft is given by,

$\mathbf{t}=\left((\mathbf{5 4 0 0} \times \mathbf{\theta}) / \boldsymbol{\pi}^{2} \mathbf{N}\right)$ seconds

Once the time period of rotation of shaft is found out we can find the angle turned by the shaft by using the formula,

$\boldsymbol{\theta}=\left(\boldsymbol{\pi}^{2} \mathrm{Nt} / \mathbf{5 4 0 0}\right)$ degrees

So, if the applied voltage is provided keeping all other values of various terms as constant the speed $\mathrm{N}$ of the motor could be found out from which the angle $\Theta$ turned by the shaft of the motor can be obtained. Hence the accelerometer provides a specific value of voltage that turns the shaft of the servomotor by a specific angle. This concept can be applied effectively for the purpose of steering in a fourwheeled automobile.

\section{CONSTRUCTION}

As discussed earlier, in an accelerometer driven servomotor based (A.S.) steering mechanism, an accelerometer is attached to the steering wheel of the automobile such that any one of the axes of the accelerometer coincides with the axis of the steering wheel. The output signals generated due to the motion of accelerometer are transferred by means of electric cables or wires. These wires originating from the output of the accelerometer terminate at the analog input ports of the microprocessor. The positive and ground wires of servomotor are directly connected to the terminals of a battery but the signal wire is connected to the output port of the microprocessor. The shaft of the servomotor is attached with the pinion, which is considered as the steering gear wheel or the steering gear cross shaft, by means of a one way coupler. The coupling takes place such that the polar axis of the shaft coincides with the axis of rotation of the pinion gear. The pinion is driven over a rack and both ends of the rack contain two knuckle arms where the middle eye of the knuckle arm is hinged about both the ends. The knuckle arms help in turning the stub axles over which the wheels are mounted about a common instantaneous centre. Electric voltage is supplied to the accelerometer as well as to the microprocessor by using voltage regulators to operate the mechanism based upon their requirement. 


\section{WORKING OF THE MECHANISM}

Inorder to operate the A.S. steering gear mechanism initially the voltage differences produced due to the motion of other two axes of accelerometer apart from the one which coincides with the steering wheel axis are fixed by not taking them into consideration and feeding the initial voltage values to the microprocessor by means of a suitable programming. Hence signal is obtained only from the change in orientation or rotation of that axis which coincides with that of the steering wheel. For the purpose of steering a vehicle three cases are considered viz. when a vehicle is moving straight, when the vehicle is taking a right turn and when the vehicle is taking a left turn.

When a vehicle is moving straight none of the wheels turn from their mean position. During this condition the accelerometer sends a constant value of signal to the microprocessor so that the microprocessor will interpret that the vehicle is needed to be moved straight. Hence the microprocessor will command the servomotor not to deviate its shaft from its mean position. It is obvious that the human hands cannot be kept steady for much time. Hence the microprocessor needs to be programmed such that for an input range of some values above or below the value at mean position the output will be generated from it that will turn the shaft of the servomotor by an angle of $0^{0}$ from its mean position. Due to no deviation in mean position of shaft the angles turned by the stub axles will be $0^{0}$. Hence the vehicle moves straight.

When a vehicle takes a right turn then the right front wheel turns inward from its mean position where as the left front wheel turns outward from its mean position. During this condition the steering wheel is to be rotated clockwise. For clockwise rotation of the steering wheel, the accelerometer generates a voltage signal due to rotation about the fixed axis. This signal is fed to the microprocessor and it is programmed such that for an input value of the range of signals generated due to clockwise rotation of the steering wheel, an output signal will be generated that will drive the motor to rotate the shaft and hence the pinion in clockwise direction. Due to clockwise rotation of pinion the rack traverses left. As a result, the right knuckle arm turns inward due to pull and left arm turns outward due to push and so does the stub axles over which wheels are mounted. Hence the vehicle goes right.

Similarly when a vehicle takes a left turn then the left front wheel turns inward from its mean position where as the right front wheel turns outward from its mean position. During this condition the steering wheel is to be rotated counterclockwise. For counter-clockwise rotation of the steering wheel, the accelerometer generates a voltage signal due to rotation about the fixed axis. This signal is fed to the microprocessor and it is programmed such that for an input value of the range of signals generated due to counterclockwise rotation of the steering wheel, an output signal will be generated that will drive the motor to rotate the shaft and hence the pinion in counter-clockwise direction. Due to counter-clockwise rotation of pinion the rack traverses right.
As a result, the right knuckle arm turns outward due to push and left arm turns inward due to pull and so does the stub axles over which wheels are mounted. Hence the vehicle goes left.

\section{THE PROGRAMMING PART}

A variety of microcontrollers along with microprocessors are available in the market. They have very good applications in various different fields like robotics and automation. Any microprocessor can be used for analog to digital conversion as well as pulse width modulation. But the logic behind any processor needs to be the same. The logic for coding the microprocessor is as follows:

- Consider the accelerometer is initially at mean position. Then the analog voltage is 2.5 volts. For this the numerical value for analog signal is 512 .

- The program is built in such manner that if the analog input voltage is $2.5 \pm 0.5$ i.e. a numerical value range of 410 to 615 then the angle of turn in the shaft of servomotor must be $0^{0}$.

- For this case the angle of turn for the corresponding voltage value is found out by using the formula $\Theta=$ $\left(\pi^{2} \mathrm{Nt} / 5400\right)$ where $\mathrm{N}$ is found out from the applied voltage $\mathrm{V}$. This value of $\Theta$ is set as mean position.

- First coding is done for ADC and then the data is manipulated for PWM output. The type of programme written for ADC to PWM conversion varies from microprocessor to microprocessor but logic behind it is same i.e. by the mapping of the analog value.

- The PWM output generated becomes the signal for the servomotor and that signal delivers the applied voltage to the servomotor.

- Hence for an applied voltage of value $\mathrm{V}$, the angle turned by shaft is $\pi^{2} \mathrm{Nt} / 5400$ ( $\mathrm{N}$ is determined from $\mathrm{V}$ ).

- When the accelerometer is rotated clockwise the voltage value increases. So, it is coded that if the analog input port receives a numerical value greater than 615 the shaft will turn by corresponding angle found out by the same formula.

- Similarly if the accelerometer is rotated anti-clockwise the voltage value decreases and proper coding is done using that formula to turn the shaft in opposite direction when the numerical value is less than 410 .

- It depends on the type of servomotor used how much maximum or minimum deviation of the shaft can be created.

\section{MERITS AND DEMERITS}

The high advantage of using an A.S. steering gear lies in the fact that a large amount of space could be reduced. Unlike a vehicle using the tradition steering gear box, where a wholesome amount of volume from the front part of the vehicle was occupied by the steering mechanism having a long rod passing through the axis of steering wheel to the steering gear cross shaft including the universal joint, there is less space used in case of this type of steering gear. Even in power steering using hydraulic actuators maximum of the space is occupied by the actuators. In case of road jerks, the 
friction is transmitted from the wheels to the hands of the driver in mechanical steering system making it uneasy for the driver. But in this kind of electronic steering system no jerks may be felt, making it smoother for the driver. Servomotors are high torque producing motors that can produce greater effort with less input. Even a medium sized servomotor would be capable of producing enough torque to steer the vehicle. No mechanical effort would be produced by the driver since the entire input system is based on electric power. Thus minimum effort is needed to be applied by the driver to produce enough torque required to steer the vehicle which is a great advantage of the A.S. steering gear over the traditional steering gear box.

However this type of steering mechanism may be more expensive than the traditional steering mechanism because of the use of servomotor along with specialized electronic components. Losses may be created due to use of a motor due to motor losses. Proper insulation would be required to avoid both heating and short circuit. In automobiles operating with petrol and diesel an additional battery pack would be required for the using this mechanism since the input system is completely based on electric power. It would be advantageous to use the A.S. steering gear in an electric vehicle (EV) since the power source for the steering system could be easily available thus making it more eco-friendly as well as user-friendly.

\section{CONCLUSION}

The rapid development in science and technology has always been beneficial for the mankind. An A.S. steering gear can make a bright future ahead in the automobile industry due to its advantageous features making it userfriendly for the driver. The driver need not have to give higher effort to steer the vehicle as in case of traditional steering gear. With proper measurement of components and proper coding it may greatly reduce the chances of skidding as well as accidents. Driving a car using the traditional steering gear would be like driving the yesterday's car where as driving a car using the A.S. steering gear would be like driving a modern car. Hence the accelerometer driven servomotor based (A.S.) steering gear will serve mankind and the society in a highly efficient manner.

\section{REFERENCES}

[1]. R. B. Gupta “Automobile Engineering”: Tech India Publication Series by Satya Prakashan

[2]. S. S. Ratan "Theory of Machines": McGraw Hill Education (India) Pvt. Ltd.

[3]. Sadhu Singh "Theory of Machines- Kinematics and Dynamics": Pearson Publications

[4]. J. B. Gupta "Theory and performance of Electrical Machines": S.K. Kataria \& Sons

[5]. https://en.wikipedia.org/wiki/Steering

[6]. https://en.wikipedia.org/wiki/Servomotor

[7]. https://www.arduino.cc/en/Tutorial/AnalogInOutSerial

[8]. https://en.wikipedia.org/wiki/Accelerometer

\section{BIOGRAPHY}

Ronauk Kumar Maharana is presently pursuing Bachelor in Technology degree in Mechanical Engineering from College of Engineering and Technology, Bhubaneswar, Odisha, India. $\mathrm{He}$ is a member of the Society of Automotive Engineers (SAE), India and presently a student co-ordinator at SAE, CET-Bhubaneswar Chapter. He is also a technical core member at Zairza Robotics Society, The Technical Club of CET-Bhubaneswar. He has completed a design course in CATIA software from Central Tool Room and Training Centre, Bhubaneswar. He can be contacted through the email address: ronauk.india@gmail.com. 